IJMMS 2004:6, 285-293

PII. S0161171204212285

http://ijmms.hindawi.com

(C) Hindawi Publishing Corp.

\title{
DUNKL WAVELETS AND APPLICATIONS TO INVERSION OF THE DUNKL INTERTWINING OPERATOR AND ITS DUAL
}

\author{
ABDELLATIF JOUINI
}

Received 28 December 2002

We define and study Dunkl wavelets and the corresponding Dunkl wavelets transforms, and we prove for these transforms Plancherel and reconstruction formulas. We give as application the inversion of the Dunkl intertwining operator and its dual.

2000 Mathematics Subject Classification: 42C15, 44A15.

1. Introduction. We consider the differential-difference operator $\Lambda_{\alpha}$ on $\mathbb{R}$ introduced by Dunkl [1] and called in the literature Dunkl operator on $\mathbb{R}$ of index $(\alpha+1 / 2)$ associated with the reflection group $\mathbb{Z}_{2}$, given by

$$
\Lambda_{\alpha} u(x)=\frac{d u(x)}{d x}+\frac{\alpha+1 / 2}{x}[u(x)-u(-x)], \quad \alpha>-\frac{1}{2} .
$$

These operators are very important in mathematics and physics. They allow the development of generalized wavelets from generalized continuous classical wavelet analysis. Moreover, we have proved in [2] that the generalized two-scale equation associated with the Dunkl operator has a solution and then we can define continuous multiresolution analysis.

Dunkl has proved in [1] that there exists a unique isomorphism $V_{\alpha}$, called the Dunkl intertwining operator, from the space of polynomials on $\mathbb{R}$ of degree $n$ onto itself, satisfying the transmutation relation

$$
\begin{gathered}
\Lambda_{\alpha} V_{\alpha}=V_{\alpha} \frac{d}{d x}, \\
V_{\alpha}(1)=1 .
\end{gathered}
$$

Rösler has proved in [3] that for each $x \in \mathbb{R}$ there exists a probability measure $\mu_{x}$ on $\mathbb{R}$ with support in the interval $[-x, x]$, such that for all polynomials $p$ on $\mathbb{R}$, we have

$$
V_{\alpha}(p)(x)=\int_{\mathbb{R}} p(y) d \mu_{x}(y) .
$$

Next, Trimèche in [5] has extended the operator $V_{\alpha}$ to an isomorphism from $\mathscr{E}(\mathbb{R})$, the space of $C^{\infty}$-functions on $\mathbb{R}$, onto itself satisfying the relations (1.1) and (1.2), and has shown that for each $x \in \mathbb{R}$, there exists a unique distribution $\eta_{x}$ in $\mathscr{E}^{\prime}(\mathbb{R})$, the space of distributions of compact support on $\mathbb{R}$, with support in the interval $[-x, x]$ such 
that

$$
\left(V_{\alpha}\right)^{-1}(f)(x)=\left\langle\eta_{x}, f\right\rangle, \quad f \in \mathscr{E}(\mathbb{R}) .
$$

He has shown also in [5] that the transposed operator ${ }^{t} V_{\alpha}$ of the operator $V_{\alpha}$ has the integral representation

$$
\forall y \in \mathbb{R}, \quad{ }^{t} V_{\alpha}(f)(y)=\int_{\mathbb{R}} f(x) d v_{y}(x), \quad f \in \mathscr{D}(\mathbb{R}),
$$

where $v_{y}$ is a positive measure on $\mathbb{R}$ with support in the set $\{x \in \mathbb{R}:|x| \geq|y|\}$ and $f$ in $\mathscr{D}(\mathbb{R})$, the space of $C^{\infty}$-functions on $\mathbb{R}$ with compact support, and ${ }^{t} V_{\alpha}$ is an isomorphism from $\mathscr{D}(\mathbb{R})$ onto itself, satisfying the relations

$$
\forall y \in \mathbb{R}, \quad{ }^{t} V_{\alpha}\left(\Lambda_{\alpha} f\right)(y)=\frac{d}{d y}{ }^{t} V_{\alpha}(f)(y),
$$

and for each $y \in \mathbb{R}$, there exists a unique distribution $Z_{y}$ in $\mathscr{Y}^{\prime}(\mathbb{R})$, the space of tempered distributions on $\mathbb{R}$, such that

$$
\left({ }^{t} V_{\alpha}\right)^{-1}(f)(y)=\left\langle Z_{y}, f\right\rangle, \quad f \in \mathscr{D}(\mathbb{R}) .
$$

In this paper, we are interested in Dunkl wavelets and associated Dunkl continuous wavelet transforms. More precisely, we give here a general construction allowing inverse formulas for the Dunkl intertwining operator and its dual.

The contents of this paper are as follows. In Section 2, we define and study the Dunkl intertwining operator and its dual. Section 3 is devoted to Dunkl wavelets and associated Dunkl wavelet transforms. In the last section, we give as application of the previous results inverse formulas for the Dunkl intertwining operator and its dual.

2. The Dunkl intertwining operator on $\mathbb{R}$ and its dual. We define and study in this section the Dunkl intertwining operator on $\mathbb{R}$ and its dual and we give their properties.

NotAtion 2.1. We have the following notations:

(i) $D_{a}(\mathbb{R})$ is the space of $C^{\infty}$-functions on $\mathbb{R}$ with support in the interval $[-a, a]$;

(ii) $S(\mathbb{R})$ is the space of $C^{\infty}$-functions on $\mathbb{R}$, rapidly decreasing together with their derivatives;

(iii) $\mathscr{Y}_{\circ}(\mathbb{R})$ is the subspace of $\mathscr{Y}(\mathbb{R})$ consisting of functions $f$ such that

$$
\forall n \in \mathbb{N}, \quad \int_{\mathbb{R}} f(x) x^{n} d x=0 ;
$$

(iv) $\mathscr{Y}_{\circ}^{\alpha}(\mathbb{R})$ is the subspace of $\mathscr{Y}(\mathbb{R})$ consisting of functions $f$ such that

$$
\forall n \in \mathbb{N}, \quad \int_{\mathbb{R}} f(x) m_{n}(x)|x|^{2 \alpha+1} d x=0,
$$

where

$$
\forall x \in \mathbb{R}, \quad m_{n}(x)=V_{\alpha}\left(\frac{u^{n}}{n !}\right)(x) ;
$$


(v) $\mu_{\alpha}$ is the measure defined on $\mathbb{R}$ by

$$
d \mu_{\alpha}(x)=\frac{|x|^{2 \alpha+1}}{2^{\alpha+1} \Gamma(\alpha+1)} d x
$$

(vi) $L_{\alpha}^{r}(\mathbb{R}), 1 \leq r \leq+\infty$, is the space of measurable functions $f$ on $\mathbb{R}$ such that

$$
\begin{aligned}
& \|f\|_{\alpha, r}=\left[\int_{\mathbb{R}}|f(x)|^{r} d \mu_{\alpha}(x)\right]^{1 / r}<+\infty, \\
& \|f\|_{\alpha, \infty}=\operatorname{ess} \sup _{x \in \mathbb{R}}|f(x)|<+\infty .
\end{aligned}
$$

DEFINITION 2.2. Define the Dunkl intertwining operator $V_{\alpha}$ on $\mathscr{E}(\mathbb{R})$ by

$$
\forall x \in \mathbb{R}, \quad V_{\alpha} f(x)= \begin{cases}\int_{-|x|}^{|x|} k_{\alpha}(x, y) f(y) d y & \text { if } x \neq 0, \\ f(0) & \text { if } x=0\end{cases}
$$

where

$$
k_{\alpha}(x, y)=\frac{\Gamma(\alpha+1)|x|^{-2 \alpha-1}}{\sqrt{\pi} \Gamma(\alpha+1 / 2)}\left(x^{2}-y^{2}\right)^{\alpha-1 / 2}(|x|+y) \chi_{]-|x|,|x|[}(y),
$$

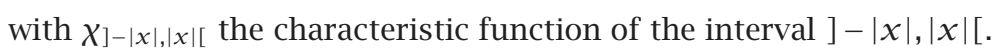

THEOREM 2.3. (i) For all $f$ in $\mathscr{E}(\mathbb{R})$,

$$
\forall x \in \mathbb{R}, \quad V_{\alpha}(f)(x)=R_{\alpha}\left(f_{e}\right)(x)+\frac{d}{d x} R_{\alpha} I\left(f_{0}\right)(x),
$$

where $f_{e}$ (resp., $f_{0}$ ) is the even (resp., odd) part of $f, R_{\alpha}$ is the Riemann-Liouville integral operator defined in [5], and $I$ is the operator given by

$$
\forall x \in \mathbb{R}, \quad I\left(f_{0}\right)(x)=\int_{0}^{|x|} f_{0}(t) d t
$$

(ii) The transform $V_{\alpha}$ is the unique topological isomorphism from $\mathscr{E}(\mathbb{R})$ onto itself, satisfying

$$
\begin{gathered}
V_{\alpha}\left(\frac{d}{d y} f\right)=\Lambda_{\alpha} V_{\alpha}(f), \quad f \in \mathscr{E}(\mathbb{R}), \\
V_{\alpha}(f)(0)=f(0) .
\end{gathered}
$$

The inverse transform $V_{\alpha}^{-1}$ is given by

$$
\forall x \in \mathbb{R}, \quad V_{\alpha}^{-1}(f)(x)=R_{\alpha}^{-1}\left(f_{e}\right)(x)+\frac{d}{d x} R_{\alpha}^{-1} I\left(f_{0}\right)(x),
$$

where $R_{\alpha}^{-1}$ is the inverse operator of $R_{\alpha}$.

Let $f$ be in $\mathscr{E}(\mathbb{R})$ and $g$ in $D(\mathbb{R})$. The operator ${ }^{t} V_{\alpha}$, defined on $D(\mathbb{R})$ by the relation

$$
\int_{\mathbb{R}} V_{\alpha}(f)(x) g(x) d \mu_{\alpha}(x)=\int_{\mathbb{R}} f(y)^{t} V_{\alpha}(g)(y) d \mu_{\alpha}(y),
$$


is given by

$$
\forall y \in \mathbb{R}, \quad{ }^{t} V_{\alpha}(f)(y)=\int_{|x| \geq|y|} k_{\alpha}(x, y) f(x) d \mu_{\alpha}(x),
$$

where $k_{\alpha}$ is the kernel given by relation (2.7). It is called the dual Dunkl intertwining operator. It has the following properties.

THEOREM 2.4. For all $f$ in $D(\mathbb{R})$,

$$
\forall y \in \mathbb{R}, \quad{ }^{t} V_{\alpha}(f)(y)={ }^{t} R_{\alpha}\left(f_{e}\right)(y)+\frac{d}{d y}{ }^{t} R_{\alpha}\left(J\left(f_{0}\right)\right)(y),
$$

where $f_{e}$ (resp., $f_{0}$ ) is the even (resp., odd) part of $f,{ }^{t} R_{\alpha}$ is the Weyl integral operator defined in [4], and $J$ is the operator given by

$$
J\left(f_{0}\right)(x)=\int_{-\infty}^{x} f_{0}(y) d y, \quad x \in \mathbb{R} .
$$

THEOREM 2.5. (i) The transform ${ }^{t} V_{\alpha}$ is a topological isomorphism from $D(\mathbb{R})$ (resp., $S(\mathbb{R})$ ) onto itself. Moreover,

$$
f \in D_{a}(\mathbb{R}) \Longleftrightarrow{ }^{t} V_{\alpha}(f) \in D_{a}(\mathbb{R}) .
$$

The inverse transform $\left({ }^{t} V_{\alpha}\right)^{-1}$ is given by

$$
\forall y \in \mathbb{R}, \quad\left({ }^{t} V_{\alpha}\right)^{-1}(f)(y)=\left({ }^{t} R_{\alpha}\right)^{-1}\left(f_{e}\right)(y)+\frac{d}{d y}\left({ }^{t} R_{\alpha}\right)^{-1}\left(J\left(f_{0}\right)\right)(y),
$$

where $\left({ }^{t} R_{\alpha}\right)^{-1}$ is the inverse operator of ${ }^{t} R_{\alpha}$.

(ii) The transform ${ }^{t} V_{\alpha}$ satisfies the transmutation relation

$$
\forall y \in \mathbb{R}, \quad{ }^{t} V_{\alpha}\left(\Lambda_{\alpha} f\right)(y)=\frac{d}{d y}{ }^{t} V_{\alpha}(f)(y), \quad f \in D(\mathbb{R}) .
$$

\section{Classical continuous wavelets and Dunkl wavelets}

3.1. Classical continuous wavelets on $\mathbb{R}$. We say that a measurable function $g$ on $\mathbb{R}$ is a classical continuous wavelet on $\mathbb{R}$ if it satisfies, for almost all $x \in \mathbb{R}$, the condition

$$
0<C_{g}^{c}=\int_{0}^{\infty}|\mathscr{F}(g)(\lambda x)|^{2} \frac{d \lambda}{\lambda}<+\infty,
$$

where $\mathscr{F}$ is the classical Fourier transform.

Let $a \in] 0,+\infty$ [ and let $g$ be a classical wavelet on $\mathbb{R}$ in $L^{2}(\mathbb{R})$. We consider the family $g_{a, x}, x \in \mathbb{R}$, of classical wavelets on $\mathbb{R}$ in $L^{2}(\mathbb{R})$ defined by

$$
g_{a, x}(y)=H_{a}(g)(x-y),
$$

where $H_{a}$ is the dilation operator given by the relation

$$
H_{a}(f)(x)=\frac{1}{a} f\left(\frac{x}{a}\right) .
$$


We define, for regular functions $f$ on $\mathbb{R}$, the classical wavelet transform $T_{g}$ on $\mathbb{R}$ by

$$
T_{g}(f)(a, x)=\int_{\mathbb{R}} f(y) \overline{g_{a, x}(y)} d y \quad \forall x \in \mathbb{R} .
$$

This transform can also be written in the form

$$
T_{g}(f)(a, x)=f * \overline{H_{a}(g)}(x),
$$

where $*$ is the classical convolution product.

The transform $T_{g}$ has been studied in [5]. Several properties are given; in particular, if we consider a classical wavelet $g$ on $\mathbb{R}$ in $L^{2}(\mathbb{R})$, we have the following results.

(i) Plancherel formula. For all $f$ in $L^{2}(\mathbb{R})$, we have

$$
\int_{\mathbb{R}}|f(x)|^{2} d x=\frac{1}{C_{g}^{c}} \int_{0}^{\infty} \int_{\mathbb{R}}\left|T_{g}(f)(a, x)\right|^{2} d x \frac{d a}{a} .
$$

(ii) Inversion formula. For all $f$ in $L^{1}(\mathbb{R})$ such that $\mathscr{F}(f)$ belongs to $L^{1}(\mathbb{R})$, we have

$$
f(x)=\frac{1}{C_{g}^{c}} \int_{0}^{\infty}\left(\int_{\mathbb{R}} T_{g}(f)(a, y) g_{a, x}(y) d y\right) \frac{d a}{a}, \quad \text { a.e., }
$$

where for each $x \in \mathbb{R}$, both the inner and the outer integrals are absolutely convergent, but possibly not the double integral.

3.2. Dunkl wavelets on $\mathbb{R}$. Using the Dunkl transform $\mathscr{F}_{D}$ and the Dunkl translation operators $\tau_{x}, x \in \mathbb{R}$, we define and study in this section Dunkl wavelets on $\mathbb{R}$ and the Dunkl continuous wavelet transform on $\mathbb{R}$, and we prove for this transform Plancherel and inversion formulas.

NotAtion 3.1. We have the following notations:

(i) $\sigma(x, y, z), \rho(x, y, z)$, and $W_{\alpha}(x, y, z)$ are the functions defined for all $x, y, z \in$ $\mathbb{R} \backslash\{0\}$ by

$$
\begin{aligned}
\sigma(x, y, z) & = \begin{cases}\frac{1}{2 x y}\left(x^{2}+y^{2}-z^{2}\right) & \text { if } x, y \neq 0, \\
0 & \text { otherwise, }\end{cases} \\
\rho(x, y, z) & =\left(\frac{1}{2}(1-\sigma(x, y, z)+\sigma(z, x, y)+\sigma(z, y, x)),\right. \\
W_{\alpha}(x, y, z) & =2^{\alpha+1} \Gamma(\alpha+1) K_{\alpha}(|x|,|y|,|z|) \rho(x, y, z),
\end{aligned}
$$

where $K_{\alpha}$ is the Bessel kernel;

(ii) for all $x, y \in \mathbb{R}, \mu_{x, y}^{\alpha}$ is the measure on $\mathbb{R}$ given by

$$
d \mu_{x, y}^{\alpha}(z)= \begin{cases}W_{\alpha}(x, y, z) d \mu_{\alpha}(z) & \text { if } x, y \neq 0, \\ \delta_{x} & \text { if } y=0, \\ \delta_{y} & \text { if } x=0,\end{cases}
$$

where $\delta_{x}$ is the Dirac measure; 
(iii) the Dunkl translation operators $\tau_{x}, x \in \mathbb{R}$, are defined on $\mathscr{E}(\mathbb{R})$ by

$$
\forall y \in \mathbb{R}, \quad \tau_{x} f(y)=\int_{\mathbb{R}} f(z) d \mu_{x, y}^{\alpha}
$$

DEFINITION 3.2. A Dunkl wavelet on $\mathbb{R}$ is a measurable function $g$ on $\mathbb{R}$ satisfying, for almost all $x \in \mathbb{R}$, the condition

$$
0<C_{g}=\int_{0}^{\infty}\left|\mathscr{F}_{D}(g)(\lambda x)\right|^{2} \frac{d \lambda}{\lambda}<+\infty
$$

where

$$
\mathscr{F}_{D}(f)(\lambda)=\int_{\mathbb{R}} f(x) \psi_{\lambda}^{\alpha}(x) d \mu_{\alpha}(x), \quad \lambda \in \mathbb{R},
$$

and $\psi_{\lambda}^{\alpha}(z)$ is the Dunkl kernel given by

$$
\psi_{\lambda}^{\alpha}(z)=\frac{\Gamma(\alpha+1)}{\sqrt{\pi} \Gamma(\alpha+1 / 2)} \int_{-1}^{1} e^{-i \lambda z t}(1-t)^{\alpha-1 / 2}(1+t)^{\alpha+1 / 2} d t, \quad \lambda, z \in \mathbb{C} .
$$

EXAMPLE 3.3. The function $\alpha_{t}, t>0$, defined by

$$
\forall x \in \mathbb{R}, \quad \alpha_{t}=\frac{C_{k}}{(4 t)^{\alpha+1 / 2}} e^{-x^{2} / 4 t},
$$

satisfies

$$
\forall y \in \mathbb{R}, \quad \mathscr{F}_{D}\left(\alpha_{t}\right)(y)=e^{-t y^{2}}
$$

The function $g(x)=-(d / d t) \alpha_{t}(x)$ is a Dunkl wavelet on $\mathbb{R}$ in $\mathscr{Y}(\mathbb{R})$, and $C_{g}=1 / 8 t^{2}$.

Proposition 3.4. A function $g$ is a Dunkl wavelet on $\mathbb{R}$ in $\mathscr{Y}(\mathbb{R})$ (resp., $\mathscr{\varphi}_{\circ}^{\circ}(\mathbb{R})$ ) if and only if the function ${ }^{t} V_{\alpha}(g)$ is a classical wavelet on $\mathbb{R}$ in $\mathscr{Y}(\mathbb{R})$ (resp., $\mathscr{Y}_{\circ}(\mathbb{R})$ ), and

$$
C_{t_{\alpha}(g)}=C_{g}
$$

Proof. The transform $\mathscr{F}_{D}$ is a topological isomorphism from $\mathscr{Y}(\mathbb{R})$ onto itself, from $\mathscr{Y}_{\circ}^{\alpha}(\mathbb{R})$ onto $\mathscr{Y}_{0}(\mathbb{R})$. We deduce then these results from Theorem 2.4.

Let $a \in] 0,+\infty$ [ and let $g$ be a regular function on $\mathbb{R}$. We consider the function $g_{a}$ defined by

$$
\forall x \in \mathbb{R}, \quad g_{a}(x)=\frac{1}{a^{2 \alpha+1}} g\left(\frac{x}{a}\right)
$$

It satisfies the following properties:

(i) for $g$ in $L_{\alpha}^{2}(\mathbb{R})$, the function $g_{a}$ belongs to $L_{\alpha}^{2}(\mathbb{R})$ and we have

$$
\mathscr{F}_{D}\left(g_{a}\right)(y)=\mathscr{F}_{D}(g)(a y), \quad y \in \mathbb{R}
$$


(ii) for $g$ in $\mathscr{Y}(\mathbb{R})$ (resp., $\left.\mathscr{Y}_{\circ}^{\alpha}(\mathbb{R})\right)$, the function $g_{a}$ belongs to $\mathscr{Y}(\mathbb{R})\left(\right.$ resp., $\left.\mathscr{Y}_{\circ}^{\alpha}(\mathbb{R})\right)$ and we have

$$
g_{a}=\left({ }^{t} V_{\alpha}\right)^{-1} o H_{a} o^{t} V_{\alpha}(g) .
$$

Let $g$ be a Dunkl wavelet on $\mathbb{R}$ in $L_{\alpha}^{2}(\mathbb{R})$. We consider the family $g_{a, x}, x \in \mathbb{R}$, of Dunkl wavelets on $\mathbb{R}$ in $L_{\alpha}^{2}(\mathbb{R})$ defined by

$$
g_{a, x}(y)=\tau_{x} g_{a}(-y), \quad y \in \mathbb{R},
$$

where $\tau_{x}, x \in \mathbb{R}$, are the Dunkl translation operators.

Using (3.15), we deduce that the family $g_{a, x}, x \in \mathbb{R}$, given by

$$
\forall y \in \mathbb{R}, \quad g_{a, x}(y)=-\tau_{x}\left(\frac{d}{d t} \alpha_{t}\right)(-y),
$$

is a family of Dunkl wavelets on $\mathbb{R}$ in $\mathscr{Y}(\mathbb{R})$.

DEFINITION 3.5. Let $g$ be a Dunkl wavelet on $\mathbb{R}$ in $L_{\alpha}^{2}(\mathbb{R})$. The Dunkl continuous wavelet transform $S_{g}^{D}$ on $\mathbb{R}$ is defined for regular functions $f$ on $\mathbb{R}$ by

$$
S_{g}^{D}(f)(a, x)=\int_{\mathbb{R}} f(y) \overline{g_{a, x}(y)}|y|^{2 \alpha+1} d y, \quad a>0, x \in \mathbb{R} .
$$

This transform can also be written in the form

$$
S_{g}^{D}(f)(a, x)=f *_{D} \overline{g_{a}}(x),
$$

where $*_{D}$ is the Dunkl convolution product defined by

$$
\forall x \in \mathbb{R}, \quad f *_{D} g(x)=\int_{\mathbb{R}} \tau_{x} f(-y) g(y) d \mu_{\alpha}(y) .
$$

THEOREM 3.6 (Plancherel formula for $S_{g}^{D}$ ). Let $g$ be a Dunkl wavelet on $\mathbb{R}$ in $L_{\alpha}^{2}(\mathbb{R})$. For all $f$ in $L_{\alpha}^{2}(\mathbb{R})$,

$$
\int_{\mathbb{R}}|f(x)|^{2}|x|^{2 \alpha+1} d x=\frac{1}{C_{g}} \int_{0}^{\infty} \int_{\mathbb{R}}\left|S_{g}^{D}(f)(a, x)\right|^{2}|x|^{2 \alpha+1} d x \frac{d a}{a} .
$$

Proof. The function $f *_{D} g$ satisfies the relation $\mathscr{F}_{D}\left(f *_{D} g\right)=\mathscr{F}_{D}(f) \cdot \mathscr{F}_{D}(g)$. Using Fubini-Tonnelli's theorem and relations (3.23) and (3.18), we obtain

$$
\begin{aligned}
\frac{1}{C_{g}} \int_{0}^{\infty} \int_{\mathbb{R}}\left|S_{g}^{D}(f)(a, x)\right|^{2}|x|^{2 \alpha+1} d x \frac{d a}{a} \\
\quad=\frac{1}{C_{g}} \int_{0}^{\infty}\left(\int_{\mathbb{R}}\left|f *_{D} \overline{\mathfrak{g}_{a}}(x)\right|^{2}|x|^{2 \alpha+1} d x\right) \frac{d a}{a}, \\
=\frac{1}{C_{g}} \int_{0}^{\infty}\left(\int_{\mathbb{R}}\left|\mathscr{F}_{D}(f)(y)\right|^{2}\left|\mathscr{F}_{D}\left(\overline{g_{a}}\right)(y)\right|^{2}|y|^{2 \alpha+1} d y\right) \frac{d a}{a}, \\
=\int_{\mathbb{R}}\left|\mathscr{F}_{D}(f)(x)\right|^{2}\left(\frac{1}{C_{g}} \int_{0}^{\infty}\left|\mathscr{F}_{D}(g)(a y)\right|^{2} \frac{d a}{a}\right)|y|^{2 \alpha+1} d y .
\end{aligned}
$$


But from Definition 3.2, we have for almost all $y \in \mathbb{R}$,

$$
\frac{1}{C_{g}} \int_{0}^{\infty}\left|\mathscr{F}_{D}(g)(a y)\right|^{2} \frac{d a}{a}=1
$$

then

$$
\frac{1}{C_{g}} \int_{0}^{\infty} \int_{\mathbb{R}}\left|S_{g}^{D}(f)(a, x)\right|^{2}\left|x^{2 \alpha+1}\right| d x \frac{d a}{a}=\int_{\mathbb{R}}\left|\mathscr{F}_{D}(f)(y)\right|^{2}\left|y^{2 \alpha+1}\right| d y .
$$

We then deduce the relation (3.25).

The following theorem gives an inversion formula for the transform $S_{g}^{D}$.

THEOREM 3.7. Let $g$ be a Dunkl wavelet on $\mathbb{R}$ in $L_{\alpha}^{2}(\mathbb{R})$. For $f$ in $L_{\alpha}^{1}(\mathbb{R})\left(\right.$ resp., $L_{\alpha}^{2}(\mathbb{R})$ ) such that $\mathscr{F}_{D}(f)$ belongs to $L_{\alpha}^{1}(\mathbb{R})$ (resp., $L_{\alpha}^{1}(\mathbb{R}) \cap L_{\alpha}^{\infty}(\mathbb{R})$ ),

$$
f(x)=\frac{1}{C_{g}} \int_{0}^{\infty}\left(\int_{\mathbb{R}} S_{g}^{D}(f)(a, y) g_{a, x}(y)|y|^{2 \alpha+1} d y\right) \frac{d a}{a}, \text { a.e., }
$$

where for each $x \in \mathbb{R}$, both the inner and the outer integrals are absolutely convergent, but possibly not the double integral.

Proof. We obtain (3.29) by using an analogous proof as for [5, Theorem 6.III.3, page 199].

4. Inversion of the Dunkl intertwining operator and of its dual by using Dunkl wavelets. Using the inversion formulas for the Dunkl continuous wavelet transform $S_{g}^{D}$ and classical continuous transform $S_{\mathfrak{g}}$, we deduce relations which give the inverse operators of the Dunkl intertwining operator $V_{\alpha}$ and of its dual ${ }^{t} V_{\alpha}$.

THEOREM 4.1. (i) Let $g$ be a Dunkl wavelet on $\mathbb{R}$ in $\mathscr{D}(\mathbb{R})$ (resp., $\mathscr{Y}(\mathbb{R})$ ). Then for all $f$ in the same space as $g$,

$$
\forall x \in \mathbb{R}, \quad S_{g}^{D}(f)(a, x)=\left({ }^{t} V_{\alpha}\right){ }^{-1}\left[S_{t_{V_{\alpha}(g)}}\left({ }^{t} V_{\alpha}(f)\right)(a, \cdot)\right](x) .
$$

(ii) Let $g$ be a Dunkl wavelet on $\mathbb{R}$ in $\varphi_{\circ}^{\circ}(\mathbb{R})$. Then for all $f$ in $\varphi_{\circ}(\mathbb{R})$,

$$
\forall x \in \mathbb{R}, \quad S_{t_{V_{\alpha}(g)}}(f)(a, x)=V_{\alpha}^{-1}\left[S_{g}^{D}\left(V_{\alpha}(f)\right)(a, \cdot)\right](x) .
$$

Proof. We deduce these results from relations (2.8), (3.22), and properties of the Dunkl convolution product.

THEOREM 4.2. Let $g$ be a Dunkl wavelet on $\mathbb{R}$ in $\varphi_{\circ}^{\alpha}(\mathbb{R})$. Then,

(i) for all $f$ in $\mathscr{Y}_{\circ}^{\circ}(\mathbb{R})$,

$$
\forall x \in \mathbb{R}, \quad S_{g}^{D}(f)(a, x)=a^{-2 \alpha} V_{\alpha}\left[S_{\mathscr{Y}\left(t V_{\alpha}(g)\right)}\left({ }^{t} V_{\alpha}(f)\right)(a, \cdot)\right](x),
$$

where $\mathscr{K}$ is the operator given by the relation

$$
\forall x \in \mathbb{R}, \quad \mathscr{K}(f)(x)=\mathscr{F}^{-1}\left[\pi \frac{|x|^{2 \alpha+1}}{2^{\alpha} \Gamma(\alpha+1)} \mathscr{F}(f)\right](x) ;
$$


(ii) for all $f$ in $\mathscr{S}_{\circ}(\mathbb{R})$,

$$
\forall x \in \mathbb{R}, \quad S_{t_{V_{\alpha}(g)}}(f)(a, x)=a^{-2 \alpha t} V_{\alpha}\left[S_{\mathscr{K}_{D}(g)}^{D}\left(V_{\alpha}(f)\right)(a, \cdot)\right](x),
$$

where $\mathscr{K}_{D}$ is the operator given by the relation

$$
\forall x \in \mathbb{R}, \quad \mathscr{K}_{D}(f)(x)=\mathscr{F}_{D}^{-1}\left[\pi \frac{|x|^{2 \alpha+1}}{2^{\alpha} \Gamma(\alpha+1)} \mathscr{F}_{D}(f)\right](x) .
$$

Proof. We obtain these relations from Theorem 4.1 and the fact that

$$
\begin{aligned}
\mathscr{K}\left({ }^{t} V_{\alpha}(g)_{a}^{0}\right) & =a^{-2 \alpha}\left(\mathscr{K}^{t}\left(V_{\alpha}(g)\right)\right)_{a}^{0}, \\
\mathscr{K}_{D}\left(g_{a}\right) & =a^{-2 \alpha}\left(\mathscr{K}_{D}(g)\right)_{a} .
\end{aligned}
$$

THEOREM 4.3. Let $g$ be a Dunkl wavelet on $\mathbb{R}$ in $\mathscr{Y}_{\circ}^{\alpha}(\mathbb{R})$. Then,

(i) for all $f$ in $\mathscr{Y}_{\circ}^{\circ}(\mathbb{R})$,

$$
\begin{aligned}
& \forall x \in \mathbb{R}, \quad\left({ }^{t} V_{\alpha}\right)^{-1}(f)(x) \\
& \quad=\frac{1}{C_{g}} \int_{0}^{\infty}\left(\int_{\mathbb{R}} V_{\alpha}\left[S_{\mathscr{K}\left(t V_{\alpha}(g)\right)}(f)(a, \cdot)\right](y) g_{a, x}(y)|y|^{2 \alpha+1} d y\right) \frac{d a}{a^{2 \alpha+1}} ;
\end{aligned}
$$

(ii) for all $f$ in $\mathscr{S}_{\circ}(\mathbb{R})$,

$$
\begin{aligned}
& \forall x \in \mathbb{R}, \quad V_{\alpha}^{-1}(f)(x) \\
& \quad=\frac{1}{C_{t V_{k}(g)}^{0}} \int_{0}^{\infty}\left(\int_{\mathbb{R}}{ }^{t} V_{\alpha}\left[S_{\mathscr{K}_{D}(g)}^{D}(f)(a, \cdot)\right](y){ }^{t} V_{\alpha}(g)_{a, x}(y) d y\right) \frac{d a}{a^{2 \alpha+1}} .
\end{aligned}
$$

Proof. We deduce (4.8) and (4.9) from Theorems 4.2, 2.4 and relation (2.9).

REMARK 4.4. We can establish in a similar way without major changes the results given above for the Dunkl intertwining operator and its dual in the multidimensional case.

\section{REFERENCES}

[1] C. F. Dunkl, Integral kernels with reflection group invariance, Canad. J. Math. 43 (1991), no. $6,1213-1227$.

[2] A. Jouini, Continuous multiresolution analysis associated with the Dunkl operator on R, Math. Sci. Res. J. 7 (2003), no. 3, 79-98.

[3] M. Rösler, Generalized Hermite polynomials and the heat equation for Dunkl operators, Comm. Math. Phys. 192 (1998), no. 3, 519-542.

[4] E. M. Stein, Interpolation of linear operators, Trans. Amer. Math. Soc. 83 (1956), 482-492.

[5] K. Trimèche, The Dunkl intertwining operator on spaces of functions and distributions and integral representation of its dual, Integral Transform. Spec. Funct. 12 (2001), no. 4, 349-374.

Abdellatif Jouini: Department of Mathematics, Faculty of Sciences, University of Tunis II, 1060 Tunis, Tunisia

E-mail address: abde11atif.jouini@fst.rnu.tn 


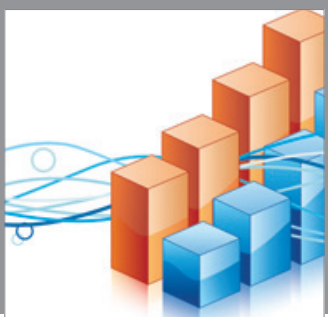

Advances in

Operations Research

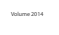

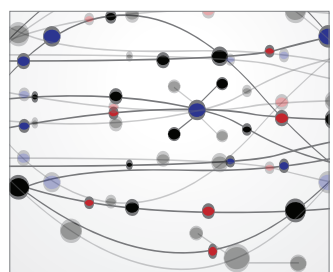

\section{The Scientific} World Journal
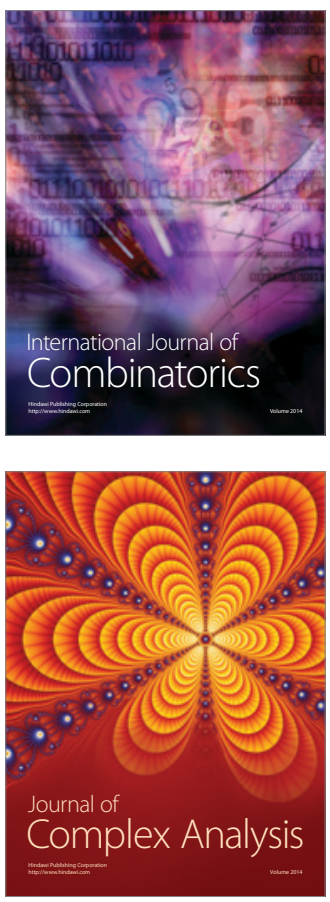

International Journal of

Mathematics and

Mathematical

Sciences
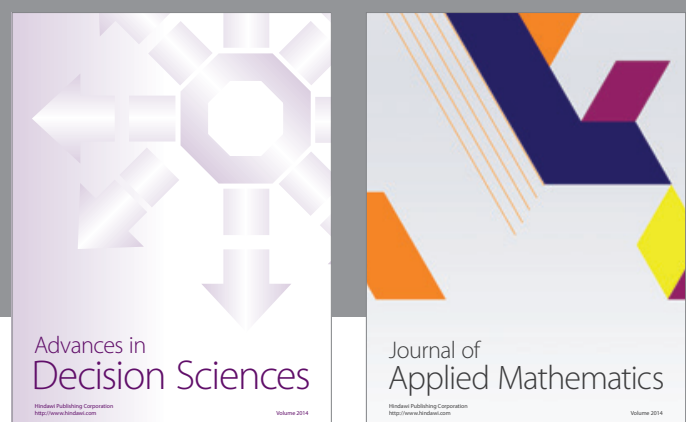

Journal of

Applied Mathematics
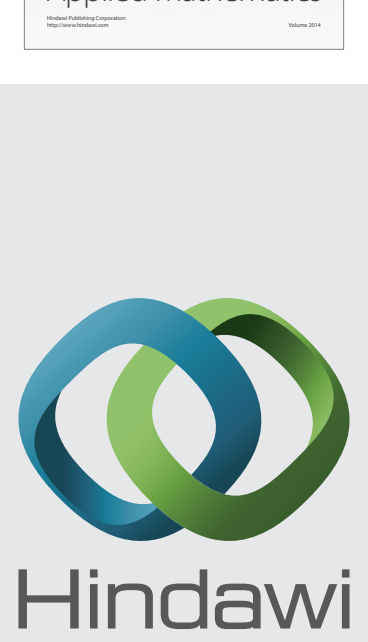

Submit your manuscripts at http://www.hindawi.com
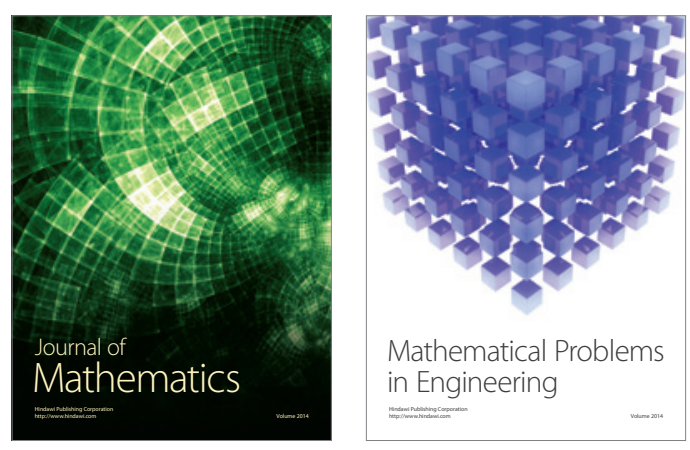

Mathematical Problems in Engineering
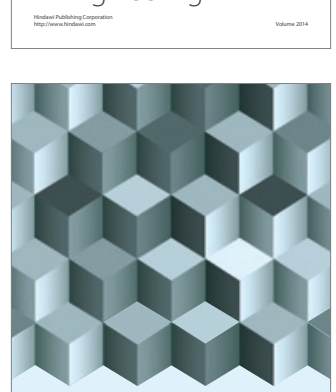

Journal of

Function Spaces
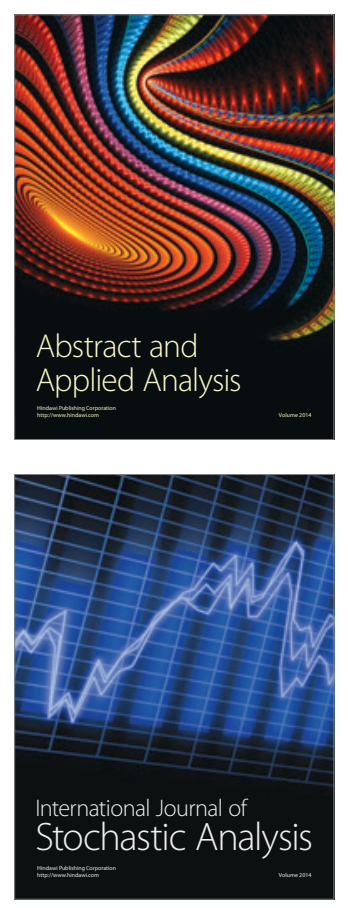

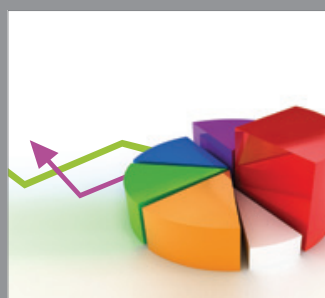

ournal of

Probability and Statistics

Promensencen
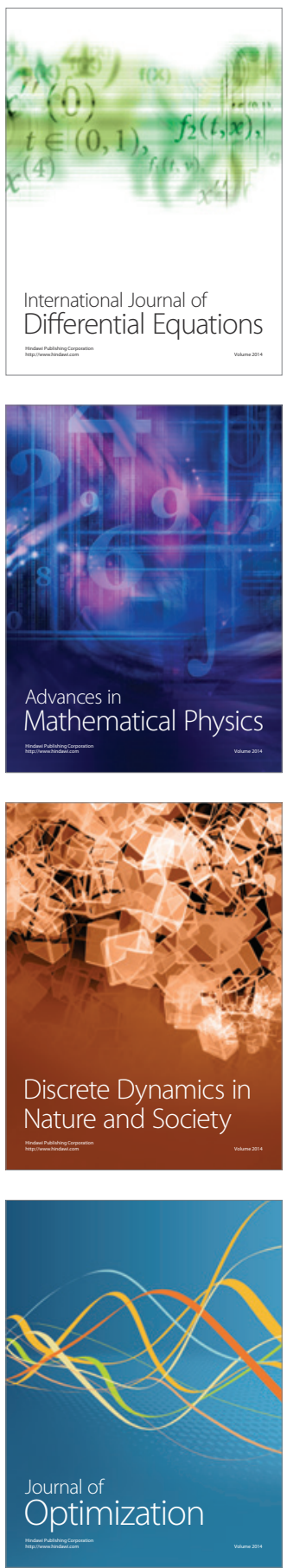